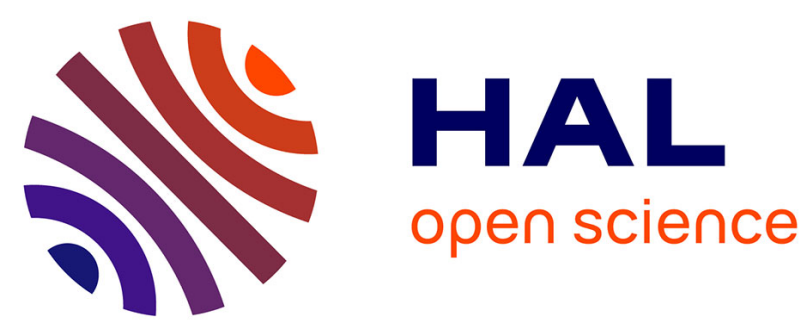

\title{
Expériences de recherche en éco-conception dans le domaine du Génie Electrique
}

\author{
Bernard Multon, Hamid Ben Ahmed, Vincent Debusschere, Yaël Thiaux, \\ Judicael Aubry, Cédric Jaouen, Franck Barruel
}

\section{To cite this version:}

Bernard Multon, Hamid Ben Ahmed, Vincent Debusschere, Yaël Thiaux, Judicael Aubry, et al.. Expériences de recherche en éco-conception dans le domaine du Génie Electrique. CONférence FRancophone sur l'Eco-conception en Génie Electrique (CONFREGE) 2010, Dec 2010, TOULOUSE, France. 8p. hal-00674884

\section{HAL Id: hal-00674884 \\ https://hal.science/hal-00674884}

Submitted on 28 Feb 2012

HAL is a multi-disciplinary open access archive for the deposit and dissemination of scientific research documents, whether they are published or not. The documents may come from teaching and research institutions in France or abroad, or from public or private research centers.
L'archive ouverte pluridisciplinaire HAL, est destinée au dépôt et à la diffusion de documents scientifiques de niveau recherche, publiés ou non, émanant des établissements d'enseignement et de recherche français ou étrangers, des laboratoires publics ou privés. 


\title{
Expériences de recherche en éco-conception dans le domaine du Génie Electrique Research experiences in eco-design in the field of Electrical Engineering
}

\author{
Bernard MULTON ${ }^{(1)}$, Hamid BEN AHMED ${ }^{(1)}$, Vincent DEBUSSCHERE ${ }^{(1)}$, Yaël THIAUX ${ }^{(1)}$, Judicaël AUBRY ${ }^{(1)}$, \\ Cédric JAOUEN ${ }^{(1,2)}$, Franck BARRUEL ${ }^{(2)}$ \\ ${ }^{(1)}$ SATIE, ENS Cachan Bretagne, CNRS, UEB, av. Robert. Schuman F-35170 Bruz \\ (2) Laboratoire des Systèmes Solaires, Institut National de l'Energie Solaire (CEA-INES), 50, avenue du Lac Léman, \\ 73377 Le Bourget du Lac
}

Mots clés : génie électrique, énergie, éco-conception, éco-dimensionnement, analyse sur cycle de vie. Key words: electrical engineering, energy, ecodesign, life cycle assessment.

\section{I- Introduction}

L'éco-conception [VENT97] consiste à concevoir des objets ou des services en considérant des critères environnementaux quantitatifs et en faisant appel à des bases de données dédiées. Cette démarche nécessite de considérer l'ensemble du cycle de vie de l'extraction de matières premières jusqu'à leur recyclage, la phase d'usage étant bien évidemment également prise en compte. L'éco-conception est souvent confondue avec l'analyse sur cycle de vie (ACV, en anglais LCA Life Cycle Assessment) [CRET05], mais ce n'est pas la même chose. Une ACV, menée généralement avec l'aide d'un logiciel spécialisé, consiste à qualifier l'objet d'étude en quantifiant une liste d'impacts environnementaux sur le cycle de vie et, le cas échéant, à proposer des pistes d'amélioration permettant de réduire des impacts considérés prioritaires., Cette phase d'amélioration peut être qualifiée de phase préliminaire d'éco-conception. La spécificité majeure de cette approche est l'aspect multi-critères compliquant vivement la formalisation d'une véritable optimisation de la phase de conception, c'est pourquoi on se satisfait généralement d'améliorations itératives obtenues à la suite d'ACV détaillées et faisant apparaître les contributions des phases de vie du produit, des différents éléments le constituant, etc.

Dans le domaine du génie électrique [MUL08], nous sommes surtout concernés par des dispositifs qui consomment ou produisent de l'énergie et dans lesquels l'impact « énergie primaire consommée sur cycle de vie » revêt une importance significative voire majeure, notamment dans le sens où de nombreux impacts (émissions de gaz à effet de serre, consommation de matières premières énergétiques non renouvelables, consommation d'eau...) ont un lien direct et prépondérant avec ce critère d'énergie primaire. En outre, nous considérons que, même si la proportion d'énergie produite à partir de ressources renouvelables augmente, l'énergie produite sera toujours perturbante pour l'environnement (bien entendu infiniment moins qu'à partir des ressources non renouvelables), l'énergie primaire consommée constituera donc sans doute toujours un critère environnemental majeur. La consommation de matières premières non énergétiques nous semble également un critère important, c'est pourquoi, les études d'éco-conception, ou plutôt d'éco-dimensionnement, que nous menons, nous ont conduits souvent à rechercher la minimisation de ces deux critères antagonistes (énergie primaire globale et masse de matériaux).

Que ce soit en génie électrique ou dans tout autre domaine, l'éco-conception est un très vaste sujet. Les travaux de recherche, que nous avons menés durant les quelques années passées et que nous poursuivons actuellement, n'en abordent qu'un volet très limité, celui que nous avons qualifié d'éco-dimensionnement. En effet, nous ne nous sommes penchés que sur la recherche de méthodologies d'optimisation de paramètres dimensionnels ou de matériaux en vue de trouver un optimum sur cycle de vie pour des produits dont nous ne remettons pas en question (pour l'instant) les procédés de fabrication, les architectures, les usages, etc.

\section{$\underline{\text { II- Importance des critères énergie primaire et ressources en matières premières }}$}

Actuellement, l'électricité contribue significativement à la dégradation de l'environnement. En 2008 [IEA10], la consommation mondiale d'énergie primaire commerciale s'élevait à environ 12,3 Gtep (142000 TWh) pour une production finale (énergie distribuée dans les circuits commerciaux) de 8,4 Gtep (98000 TWh). Plus de 87\% des ressources primaires utilisées étaient d'origine non renouvelable (fossiles et fissiles). En ce qui concerne l'électricité, près de $40 \%$ de l'énergie primaire totale a été consommée pour produire $20200 \mathrm{TWh}_{\mathrm{e}}$ dont 16700 ont été commercialisés, ce qui donne un rendement global d'environ 30\% incluant les pertes dans les réseaux. La figure 1 montre le bilan global mondial 2008 (données issues de [IEA10]) et la place de l'électricité dans ce bilan.

La figure 2 [OBS09] montre, quant à elle, la répartition des sources de production d'électricité. La proportion de ressources renouvelables y est plus élevée $(18,7 \%)$ et si l'on considérait les taux de croissance [OBS09] des différents moyens de production, on pourrait constater que la part des renouvelables croit beaucoup plus vite que celle des non renouvelables. En ajoutant le fait que la part de l'électricité dans le bilan final mondial est également en croissance, on peut constater que l'électricité possède un fort potentiel de contribution au développement durable.

En ce qui concerne les ressources et réserves en matières premières non énergétiques, nous avons récapitulé, dans le tableau 1, des données 2006 de l'USGS (U.S. Geological Survey) concernant quelques matériaux importants dans le secteur électrique. Le rapport réserves sur production primaire (non issue des filières de recyclage) met en évidence leur rareté et les tensions sur les marchés. 


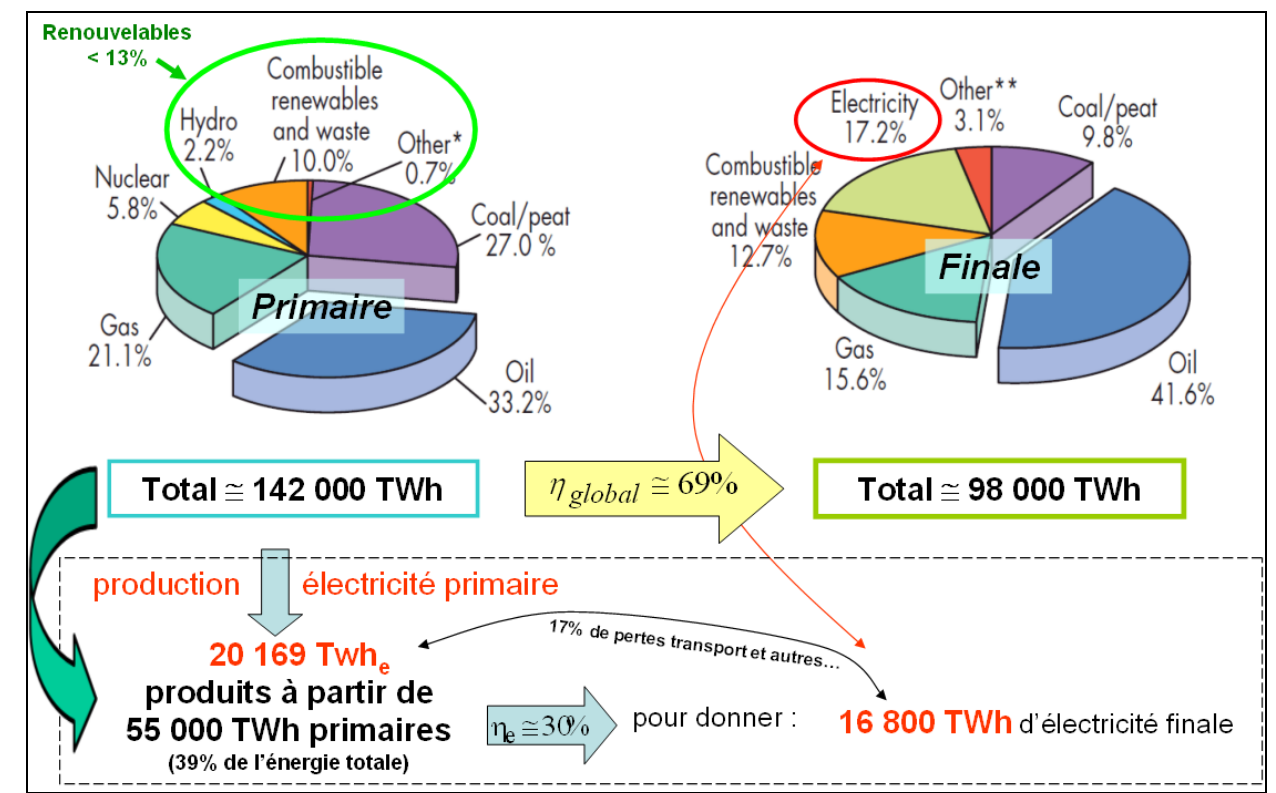

Figure 1 : bilan énergétique mondial 2008 (données issues de [IEA10]). World Energy Balance 2008 (data from [IEA10])

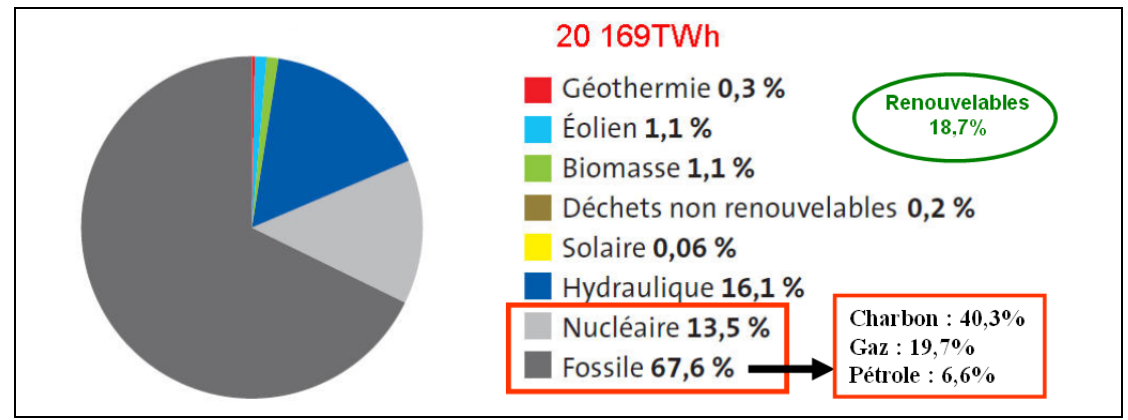

Figure 2 : sources pour la production mondiale d'électricité 2008 (données issues d'Observ'ER [OBS09]). Sources for world electricity production in 2008 (data from Observ'ER [OBS09])

\begin{tabular}{|c|c|c|c|c|c|c|}
\hline \multirow{2}{*}{ En tonnes } & \multirow{2}{*}{$\begin{array}{l}\text { Ressources } \\
\text { présumées }\end{array}$} & \multirow{2}{*}{$\begin{array}{c}\text { Réserves } \\
\text { Base }\end{array}$} & \multirow{2}{*}{ Réserves } & \multicolumn{2}{|c|}{ Production Mondiale 2006 } & $\begin{array}{c}\text { Réserves/Product. } \\
\text { primaire } \\
\text { (ans) }\end{array}$ \\
\cline { 5 - 7 } & primaire & secondaire & 40 \\
\hline Acier & $230 \mathrm{G}$ & $160 \mathrm{G}$ & $73 \mathrm{G}$ & $1,8 \mathrm{G}$ & & 167 \\
\hline $\mathbf{A l u}$ & 13 à $18 \mathrm{G}$ & $8 \mathrm{G}$ & $5,7 \mathrm{G}$ & $34 \mathrm{M}$ & $7,6 \mathrm{M}(2004)$ & 31 \\
\hline $\mathbf{C u}$ & 3 à $3,7 \mathrm{G}$ & $940 \mathrm{M}$ & $470 \mathrm{M}$ & $15 \mathrm{M}$ & & 17 \\
\hline $\mathbf{P b}$ & $1,5 \mathrm{G}$ & $140 \mathrm{M}$ & $67 \mathrm{M}$ & $4 \mathrm{M}$ & $4 \mathrm{M}$ & 122 \\
\hline $\mathbf{C o}$ & $15 \mathrm{M}$ & $13 \mathrm{M}$ & $7 \mathrm{M}$ & $57,5 \mathrm{k}$ & & 46 \\
\hline $\mathbf{N i}$ & $130 \mathrm{M}$ & $140 \mathrm{M}$ & $64 \mathrm{M}$ & $1,4 \mathrm{M}$ & & 156 \\
\hline $\mathbf{P t}$ & $76 \mathrm{k}$ & & $33 \mathrm{k}$ & 211 & 50 & 218 \\
\hline $\mathbf{L i}$ (Metal) & $14 \mathrm{M}$ & $11 \mathrm{M}$ & $4,1 \mathrm{M}$ & $18,8 \mathrm{k}$ & & \\
\hline
\end{tabular}

Tableau 1 : bilan des ressources et réserves 2006 (données issues de l'U.S.G.S.).

Stock of resources and reserves in 2006 (data from USGS).

Au-delà des impacts environnementaux liés à l'extraction des matières premières (énergétiques on non) et à leurs traitements, leurs prix sont très fluctuants et affectent considérablement l'économie mondiale.

Sur la base des niveaux de consommation mondiale et des prix suivants :

- 4 Gtep pétrole / an => $2300 \mathrm{G} \$$ (pétrole à $80 \$ /$ baril soit $600 \$ /$ tep),

- 3,2 Gtep charbon /an => $500 \mathrm{G} \$$ (charbon à $150 \$ /$ tep),

- 2,5 Gtep gaz /an $=>400 \mathrm{G} \$($ gaz à $150 \$ /$ tep$)$,

- 0,7 Gtep uranium / an => $10 \mathrm{G} \$$ ((uranium à $60 \$ /$ livre),

On arrive à une dépense mondiale annuelle en matières premières énergétiques non renouvelables de $3200 \mathrm{G} \$$ soit $5,5 \%$ du PIB mondial. On comprend ainsi la très grande sensibilité de notre économie aux fluctuations des cours et à leur inévitable dérive à la hausse. Il en va de même pour les dépenses associées aux matières premières non énergétiques dont nous n'avons pas fait de bilan ici.

Les problèmes de développement durable associés concernent bien sûr l'environnement, mais également les limites de ressources et, en fin de compte, l'économie mondiale. 


\section{III-Problèmes associés à l'éco-dimensionnement de dispositifs et systèmes électriques}

La variété des problèmes est grande et nous nous garderons, au stade actuel de notre expérience, de généraliser à l'excès notre vision.

Jusqu'à maintenant, l'expérience cumulée a concerné :

- des objets consommateurs d'énergie, par exemple un moteur électrique

- des systèmes de production d'énergie, par exemple un système photovoltaïque autonome

- des systèmes de distribution d'électricité, l'objectif étant ici d'évaluer l'intérêt de la distribution en courant continu dans les bâtiments.

Pour ce dernier cas, l'approche cycle de vie nous a semblé constituer une voie pertinente pour évaluer plus rationnellement l'intérêt de la distribution en courant continu dans les bâtiments, en comparaison avec celle, traditionnelle, en courant alternatif, notamment pour rechercher l'influence du niveau de tension.

Nous proposons, à travers quelques exemples, de montrer les spécificités de quelques unes de nos études. L'organigramme de la figure 3 met en évidence les principaux blocs de la procédure d'éco-dimensionnement telle que nous la voyons.

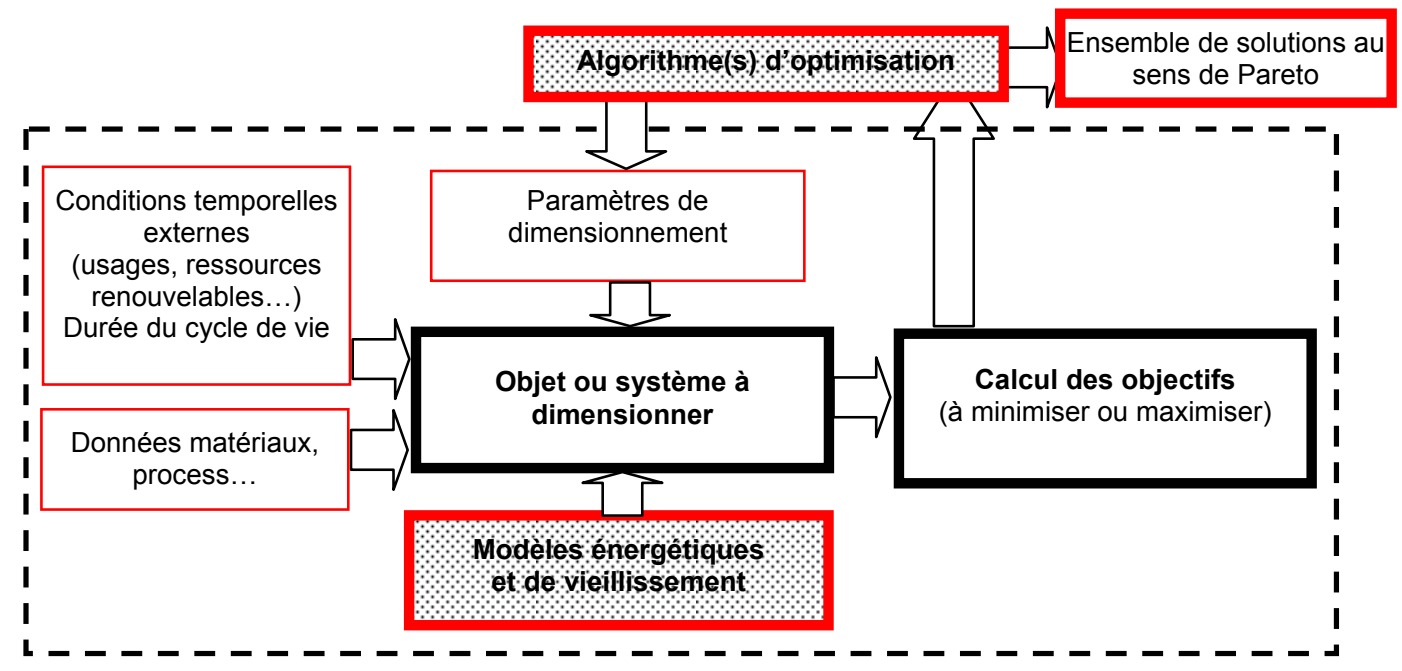

Figure 3 : organigramme d'éco-dimensionnement.

Eco-sizing diagram.

Le traitement de ce problème nécessite de porter les efforts sur :

- l'établissement de modèles énergétiques aptes au dimensionnement sur des cycles temporels éventuellement longs et complexes, c'est-à-dire prenant en compte les spécificités physiques pour le calcul des consommations d'énergie et les contraintes de temps de calcul ;

- l'établissement et/ou l'exploitation de modèles de durée de vie permettant de bien optimiser le dispositif sur la durée totale de son cycle de vie ;

- l'utilisation et/ou la mise au point d'algorithmes d'optimisation aptes à traiter de tels problèmes ;

- l'obtention de données environnementales (ici l'énergie primaire requise pour la production des matériaux utilisés, pour les process de fabrication et de recyclage et, le cas échéant, pour les phases de transport).

En comparaison avec une optimisation «classique» de dimensionnement d'un dispositif électrique sur cycle de fonctionnement, et non sur un seul point dimensionnant (ou nominal), la différence majeure réside dans le choix des critères et dans le couplage aux modèles de vieillissement. Notons que ce que nous avons qualifié d'optimisation " classique » sur cycle constitue une famille de problèmes également loin d'être correctement résolus, surtout lorsque l'on considère une échelle plus système. La référence [AUB10a] montre d'ailleurs les difficultés de l'optimisation (ici critère économique) du dimensionnement d'un ensemble convertisseur machine (dimensionnements fortement couplés du convertisseur électronique de puissance et de la machine) sur cycle (sans couplage aux lois de vieillissement).

Le couplage aux lois de vieillissement nécessite de connaître au préalable les mécanismes de défaillance les plus influents, ce qui constitue déjà un point difficile. Dans les travaux que nous avons menés, nous avons entre autres considérés les vieillissements :

- thermique (cas des isolants des moteurs, condensateurs ou supercondensateurs),

- électrique (condensateurs ou supercondensateurs)

- électrochimique (accumulateurs électrochimiques, modèles de dégradation en cyclage, sans prise en compte des effets de la température)

- photovoltaïque.

Le problème du vieillissement lui-même peut d'ailleurs être traité de différentes façons ;

- « simple » limite de durée de vie associée à la grandeur physique impactante, 
- $\quad$ prise en compte de la dégradation de caractéristiques essentielles (par exemple la capacité énergétique de stockage d'un accumulateur),

- $\quad$ vision probabiliste par prise en compte des taux de défaillance [LIS10].

Finalement, l'éco-optimisation constitue un problème similaire à celui d'un dimensionnement sur critères purement économiques. C'est d'ailleurs la démarche que nous avons adoptée pour optimiser, sur cycle de vie et sur critères économiques, la gestion d'énergie et le dimensionnement d'un système de stockage à supercondensateurs pour le lissage de la production d'énergie d'un houlogénérateur [AUB10b]. Pour ce cas d'étude, la loi de vieillissement considérée combine les effets de la tension et de la température [DIA06].

Pour ne pas rester plus longtemps sur des généralités, nous proposons de donner quelques exemples de problèmes traités ou en cours de traitement par notre équipe de recherche.

\section{$\underline{\text { IV-Expériences d'éco-dimensionnement en génie électrique }}$}

\section{IV.1- Transformateur monophasé : un cas d'école pour considérer les aspects thermiques transitoires et le} vieillissement [DEB09]

Il s'agit ici d'un cas d'école, un transformateur monophasé alimenté à tension et fréquence fixes, permettant de poser le problème d'une optimisation dans des situations de complexité croissante. Un algorithme génétique évolutionnaire est utilisé pour l'optimisation. Les objectifs à minimiser sont l'énergie primaire cumulée sur cycle de vie (GER = Gross Energy Requirement) et la masse totale des parties actives (circuit magnétique et bobinage), pour la fourniture de $200 \mathrm{VA}$ pendant 5 heures par jour pendant 7 ans. En notant $\mathrm{E}_{\mathrm{m}, \mathrm{p}}$ l'énergie primaire élémentaire consommée par un kilogramme du matériau $m$ lors de la phase $p$ de son cycle de vie et $\mathrm{P}_{\text {fct }}$ l'ensemble des pertes cumulées lors du fonctionnement du transformateur, nous avons l'expression du GER suivante :

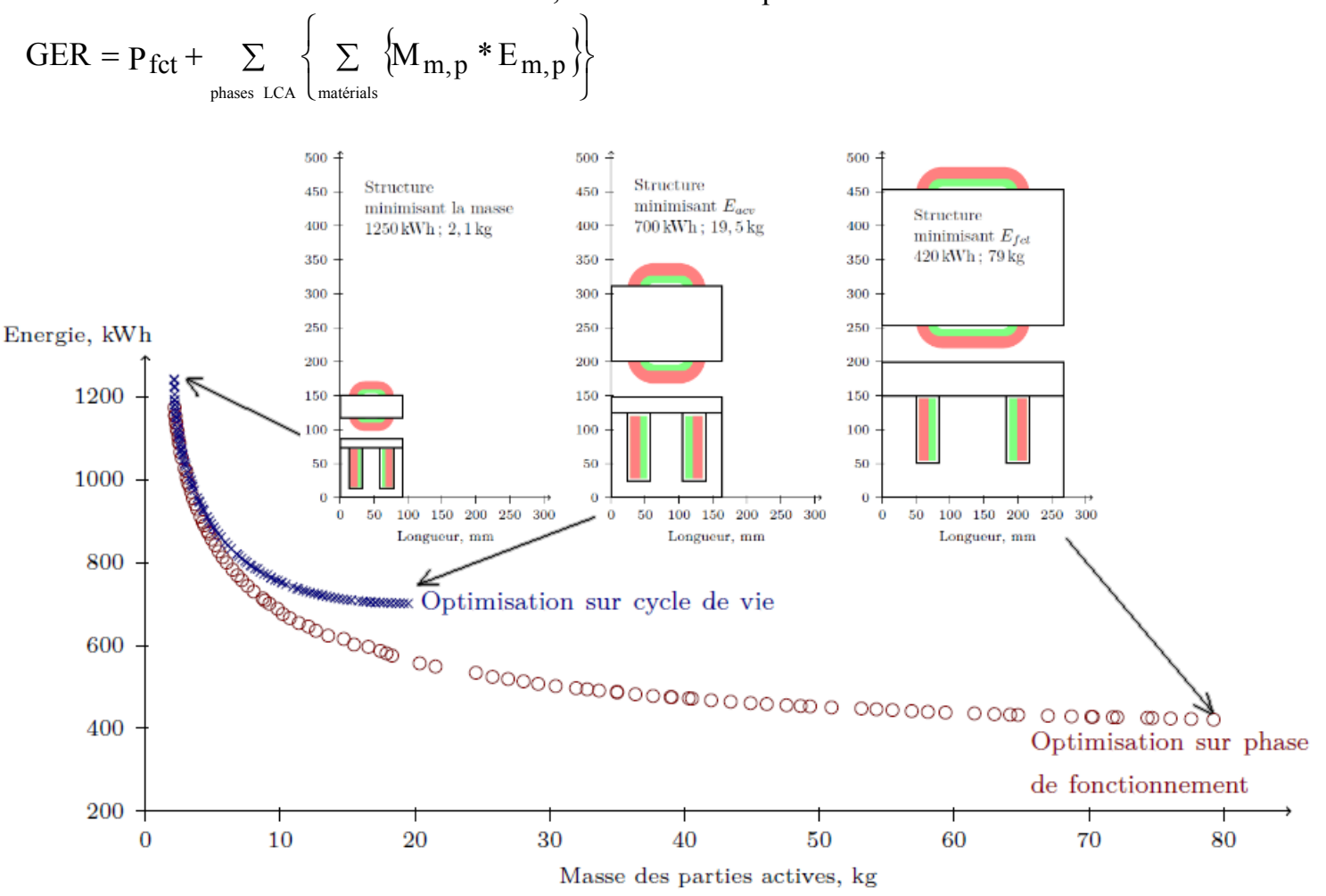

Figure 4 : paramètres du transformateur et fronts de Pareto obtenus.

Transformer parameters and Pareto Front results.

Les résultats présentés à la figure 4 montrent un premier résultat d'optimisation des dimensions et du nombre de spires sans prise en compte du vieillissement et en supposant le régime permanent thermique atteint instantanément. On peut y voir tout d'abord les solutions obtenues dans une optimisation «classique » avec minimisation des seules pertes de fonctionnement, les solutions extrêmes correspondent aux limites d'échauffement (masse minimale) et aux limites dimensionnelles autorisées (masse maximale). Sur le même graphique sont également représentées les solutions obtenues avec une optimisation sur cycle de vie incluant la dépense d'énergie pour la production des matériaux (précisons que l'énergie utile n'intervient pas dans le bilan GER). On y remarque une solution optimale de masse intermédiaire correspondant à un compromis entre pertes énergétiques cumulées et investissement énergétique. Pour une masse très faible, le GER calculé sur le cycle de fonctionnement est très proche des pertes intégrées sur la durée d'usage, le coût énergétique lié à la matière première devenant négligeable. A l'inverse, choisir un transformateur plus 
gros ne conduirait pas à une diminution suffisante de ses pertes de fonctionnement comparativement au surcoût d'investissement énergétique. Par ailleurs, si on avait choisi une durée de fonctionnement plus courte, la solution optimale sur cycle de vie aurait été de plus faible masse car le poids énergétique des pertes aurait régressé par rapport au cas précédent. C'est ce qu'illustre la figure 5 montrant les effets de la durée de fonctionnement $(2,5$ à $10 \mathrm{~h}$ par jour $)$ dans les conditions précédentes. On constate bien que la masse optimale diminue avec la durée de fonctionnement.

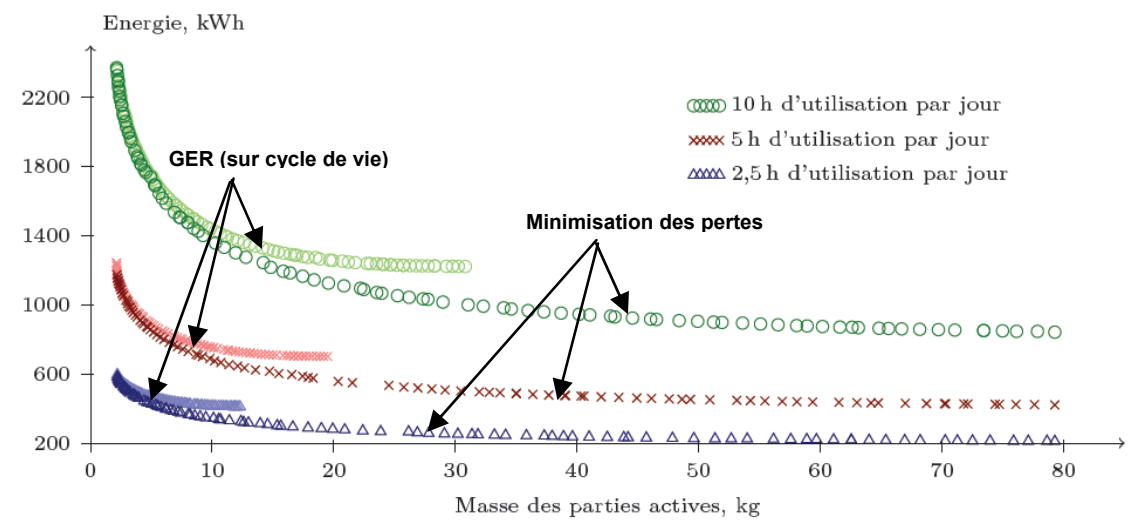

Figure 5 : effet de la durée de fonctionnement sur les solutions optimales minimisant le GER ou les pertes. Effect of operating time on the optimal solutions minimizing the GER or only losses.

Si l'on considère des durées de fonctionnement courtes (ou des variations rapides de la puissance) devant la constante de temps thermique, les échauffements seront en réalité moins élevés que ceux déterminés par la modélisation en régime thermique permanent. C'est la raison pour laquelle il est nécessaire d'inclure une modélisation thermique en régime transitoire dans les cas de fonctionnements fortement variables ou relativement impulsionnels. Des simulations temporelles sont alors requises ce qui conduit à un allongement sensible des temps de résolution. Dans ce cas, la seule zone du front de Pareto modifiée est celle des faibles masses qui peut alors être étendue vers la gauche, sauf si l'on avait considéré le couplage des propriétés physiques (résistivité, coefficients de pertes magnétiques) avec la température, ce qui aurait modifié le calcul des pertes. Ces simulations temporelles permettent éventuellement d'atteindre des valeurs optimales précédemment non accessibles. Ce sera le cas avec le moteur électrique exemple d'application de la section suivante.

La méthode permet également d'analyser la sensibilité aux caractéristiques environnementales des matériaux et au mode de production d'électricité (prise en compte du rendement). En effet, les caractéristiques environnementales (ici l'énergie primaire requise par unité de masse) dépendent fortement des process de fabrications, de l'origine des matières premières (concentration du minerai, proportion de matière issue du recyclage, distances de transport...). On peut ainsi considérer des données pour une zone géographique particulière ou pour le monde entier. En outre, les évolutions technologiques conduisent plutôt à une tendance aux améliorations des impacts. Il est également possible d'étudier la sensibilité à des matériaux alternatifs, par exemple l'aluminium à la place du cuivre.

Dernier point de cette étude : l'éco-dimensionnement avec couplage aux lois de vieillissement. Nous considérons ici la loi classique de dégradation de la durée de vie avec la température (« loi de Monsinger ») :

$\mathrm{T}_{\mathrm{i}}=\mathrm{T}_{\text {ref }} \cdot 2^{\frac{\theta_{\text {ref }}-\theta_{\mathrm{i}}}{6}}$ avec, par exemple pour un isolant de classe $\mathrm{B}, \theta_{\text {ref }}=130^{\circ} \mathrm{C}$ et $\mathrm{T}_{\text {ref }}=20000 \mathrm{~h}$

Cette considération permet, sur une durée d'usage donnée, d'envisager la possibilité de remplacements du produit et donc une comptabilisation d'un investissement énergétique cumulant plusieurs exemplaires du même produit. Nous avons alors observé la possibilité d'étendre le front de Pareto à gauche (solutions à plus faible masse et de plus grande énergie cumulée sur cycle de vie) comme dans le cas de la prise en compte des régimes transitoires thermiques [DEB09].

\section{IV.2- Moteur électrique [DEB09]}

Le moteur concerné ici est de type asynchrone monophasé (diphasé à condensateur). Il est destiné à un actionneur de volet roulant. Le réducteur de vitesse auquel il est associé ainsi que tout le système mécanique sont imposés. Même le profil da découpe des tôles est défini, les seuls paramètres disponibles sont la longueur active (associée à la masse), le nombre de spires du bobinage et le condensateur de déphasage. L'alimentation est directe $(230 \mathrm{~V}-50 \mathrm{~Hz})$, elle est monophasée avec condensateur permanent de déphasage, le circuit magnétique est fondamentalement saturé et le régime thermique est fondamentalement transitoire. Le fait qu'il s'agisse d'une machine asynchrone, dont la vitesse ne varie pas beaucoup en fonction du couple, a permis de découpler le comportement du moteur et celui de la charge mécanique. Ainsi, le cycle de fonctionnement (une montée et une descente) est supposé imposé par la charge : un couple résistant qui varie presque sinusoïdalement en fonction du temps.

La courte durée des cycles et la faible taille du moteur (sur toute la plage des longueurs permise) permettent de considérer un échauffement adiabatique et donc de simplifier considérablement le traitement de l'échauffement sachant 
que le problème de dimensionnement est contraint par une température maximale, ce qui constituera la limite basse de longueur active.

En outre, la petite taille de ce moteur et son faible rendement lui confèrent, bien qu'il soit saturé, un comportement relativement résistif avec une impédance qui varie très peu en fonction du couple, ceci nous a permis de considérer des courants d'alimentation sinusoïdaux et toujours déphasés de $\pi / 2$, ce qui a été vérifié expérimentalement.

Ceci a permis de simplifier considérablement l'étude et la quantité de calculs par éléments finis (en magnétodynamique non linéaire) [DEB10b]. Nous avons entre autres mis en évidence l'existence d'un optimum de longueur active (et donc de quantité de matériaux) pour minimiser l'énergie primaire consommée sur cycle de vie alors que la recherche d'un maximum de rendement sur cycle de fonctionnement conduisait à maximiser la quantité de matière active pour minimiser les pertes électriques (Joule et magnétiques).

Nous avons également établi la notion de rendement sur cycle de vie correspondant aux usages du dispositif :

$$
\eta_{\text {lca }}=\frac{\int \mathrm{P}_{\mathrm{u}}(\mathrm{t}) / \eta_{\mathrm{e}} \cdot \mathrm{dt}}{\int_{\text {life }} \mathrm{P}_{\text {cons }}(\mathrm{t}) / \eta_{\mathrm{e}} \cdot \mathrm{dt}+\mathrm{W}_{\mathrm{fab}+\text { recycl_p }}}=\frac{\mathrm{W}_{\mathrm{u}_{-} \mathrm{p}_{-} \text {life }}}{\mathrm{W}_{\mathrm{u}_{-} \mathrm{p}_{-} \text {life }}+\left[\mathrm{W}_{\text {losses__ } \mathrm{p}_{-} \text {life }}+\mathrm{W}_{\text {fab+recycl_p }}\right]}
$$

où $\mathrm{P}_{\mathrm{u}}(\mathrm{t})$ est la puissance utile, $\eta_{\mathrm{e}}$ le rendement de production d'électricité (pour déterminer l'énergie primaire consommée), $\mathrm{P}_{\text {cons }}(\mathrm{t})$ la puissance consommée (utile+pertes) durant la phase de fonctionnement du composant et $\mathrm{W}_{\text {fab+recycl_p }}$ l'énergie primaire de fabrication et de recyclage.

La figure 6 montre quelques résultats, l'énergie primaire consommée correspond à la part $\mathrm{W}_{\text {losses_p_life }}+\mathrm{W}_{\text {fab+recycl_p }}$ sachant que l'énergie utile $\left(\mathrm{W}_{\mathrm{u} \_ \text {p_life }}\right)$ ne varie pas à usage donné. On peut remarquer sur la figure de droite l'existence d'un rendement sur cycle de vie optimal alors qu'avec une approche limitée à la seule phase de fonctionnement, le rendement électrique s'améliore continument avec la masse. Les deux rendements, sur cycle de vie et sur cycle de fonctionnement, se distinguent d'autant plus que l'usage est bref par rapport à la durée de vie du produit. Ainsi, un usage plus intense (ici 5 cycles par jour) justifie une masse active plus importante pour atteindre un rendement sur cycle de vie maximal plus élevé.
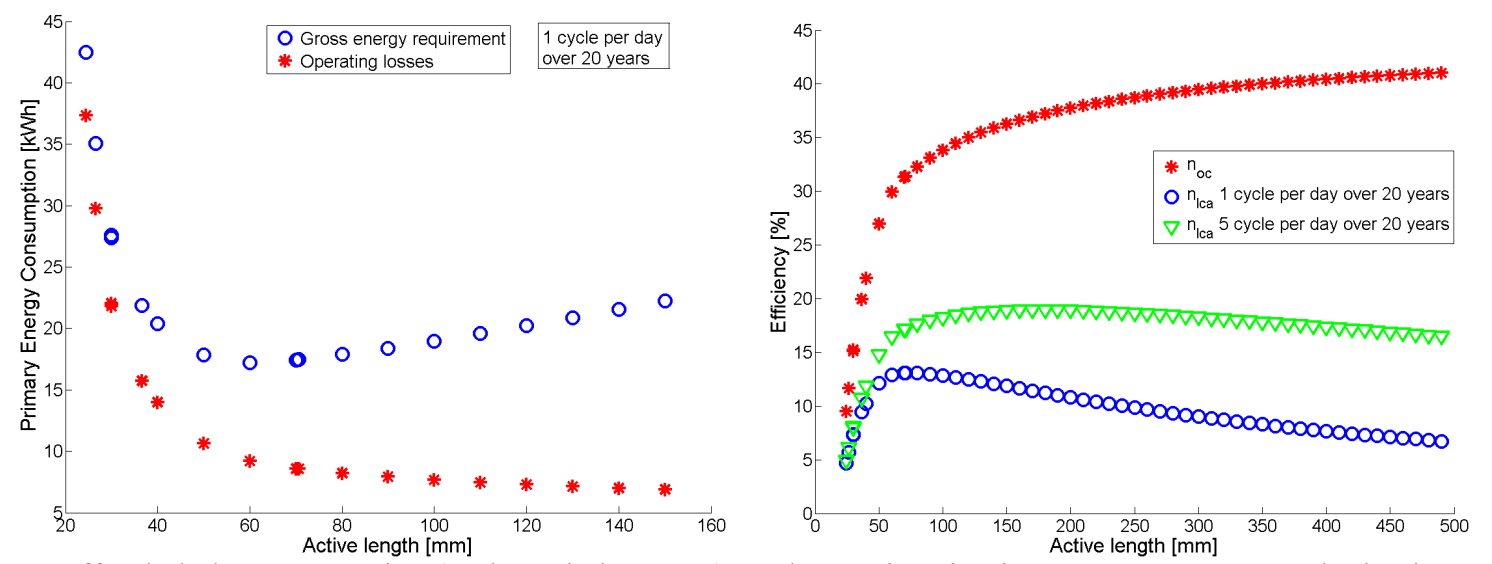

Figure 6 : effet de la longueur active (et donc de la masse) sur l'énergie primaire consommée sur cycle de vie et sur les pertes de conversion (à gauche). Rendements (à droite) sur un cycle de montée-descente $\left(\eta_{\text {oc }}\right)$ et sur cycle de vie ( $\eta_{\text {lca }}$ ) en fonction de l'usage.

Effect of the active length (and so the mass) on the primary energy consumed on life cycle and losses (left). Efficiency curves (right) on only one operating cycle $\left(\eta_{e}\right)$ and life cycle $\left(\eta_{\text {lca }}\right)$ depending on use.

\section{IV.3- Système photovoltaïque autonome [THI10b]}

Nous considérons ici un système photovoltaïque avec accumulateur électrochimique et convertisseurs (hacheur à contrôle MPPT, permettant notamment des délestages progressif de production, et onduleur). L'un des objectifs consiste dans l'évaluation de l'intérêt de la gestion des profils de consommation sur la réduction de l'énergie primaire consommée sur cycle de vie. Celle-ci est déterminée par les investissements énergétiques incluant les remplacements éventuels de l'accumulateur, la durée de vie du système étant définie par celle du générateur PV. L'autre objectif majeur concerne la comparaison des technologies d'accumulateur plomb-acide et lithium-ion sur le coût énergétique sur cycle de vie de l'ensemble du système.

Cette optimisation de dimensionnement consiste à déterminer les valeurs optimales de la capacité énergétique et de la puissance nominale installée photovoltaïque dans un contexte de données de rayonnement solaire et de consommation déterministes. Nous avons choisi deux critères contradictoires, l'énergie primaire consommée sur l'ensemble du cycle de vie (GER) du système, incluant les remplacements éventuels de l'accumulateur, et le taux de non fourniture du consommateur (lorsqu'il est nul, la totalité de ses besoins sont satisfaits).

A même énergie fournie au consommateur sur la durée d'usage, nous avons pu montrer la très grande influence du profil de consommation et les gains très significatifs envisageables sur le GER. Nous avons également constaté que, même avec les technologies actuelles (silicium polycristallin et accumulateurs plomb-acide ou lithium-ion) et avec le niveau de rayonnement solaire de la région rennaise, le GER était, dans tous les cas, inférieur à celui du système 
électrique traditionnel, en ne comptabilisant d'ailleurs que la phase de production d'électricité. Ces résultats ont déjà été publiés, notamment dans [THI10a], et nous proposons ici de montrer l'incidence de la prise en compte du vieillissement (dégradation des caractéristiques du générateur PV et de l'accumulateur) sur le dimensionnement du système. La figure 7 [THI10b] montre le front de Pareto obtenu par optimisation minimisant le GER et le taux de non fourniture du consommateur (LLP = Loss of Load Probability). Les lois de vieillissement employées sont simplistes (dégradation continue dans le temps de la puissance nominale $\mathrm{PV}$ et de l'accumulateur au plomb associée à son cyclage sachant que son remplacement est effectué lorsque sa capacité de stockage atteint $80 \%$ de sa capacité initiale). On remarquera que cette prise en compte conduit à un surcoût énergétique (et économique) sur l'ensemble du cycle de vie d'environ $20 \%$, ce qui est non négligeable.
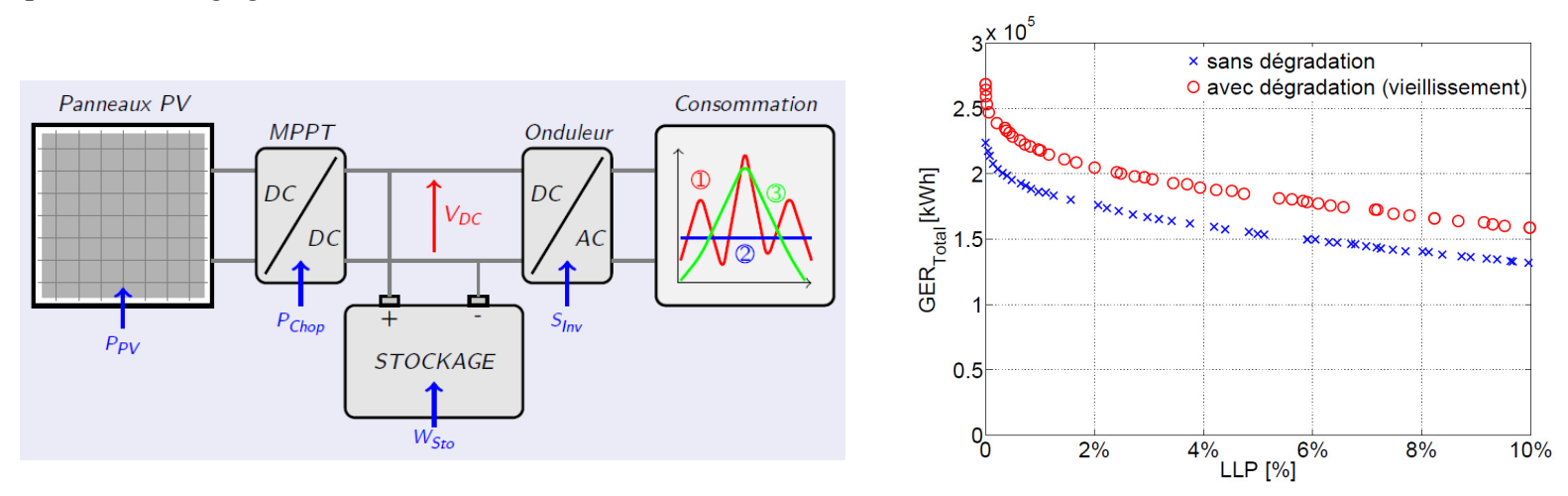

Figure 7 : Système PV autonome et effet de la prise en compte de lois de vieillissement sur le GER d'un système photovoltaïque autonome [THI10b].

Stand-Alone PV system and Effect of taking into account of aging laws on the GER of an autonomous photovoltaic system [THI10b].

\section{IV.4- Réseau de distribution électrique dans un bâtiment}

Il s'agit ici d'un travail encore plus exploratoire destiné à proposer un critère objectif pour l'étude de l'intérêt d'un passage à une distribution en courant continu dans des bâtiments résidentiels ou tertiaires. En effet, l'argument généralement avancé consiste à mettre en avant les pertes dans les multiples conversions requises par l'alimentation traditionnelle en courant alternatif pour alimenter la plupart des appareils électriques modernes. Mais l'alimentation directe sous tension continue nécessitera aussi de toute façon des convertisseurs (DC-DC) dont les pertes dépendront des choix de dimensionnement de leurs composants. En outre, il sera nécessaire d'optimiser le niveau de la tension distribuée. Nous posons donc le problème de la minimisation du GER par l'optimisation du dimensionnement, d'une part, du câblage du réseau de distribution, en fonction du niveau de tension et des usages, et d'autre part des convertisseurs statiques.
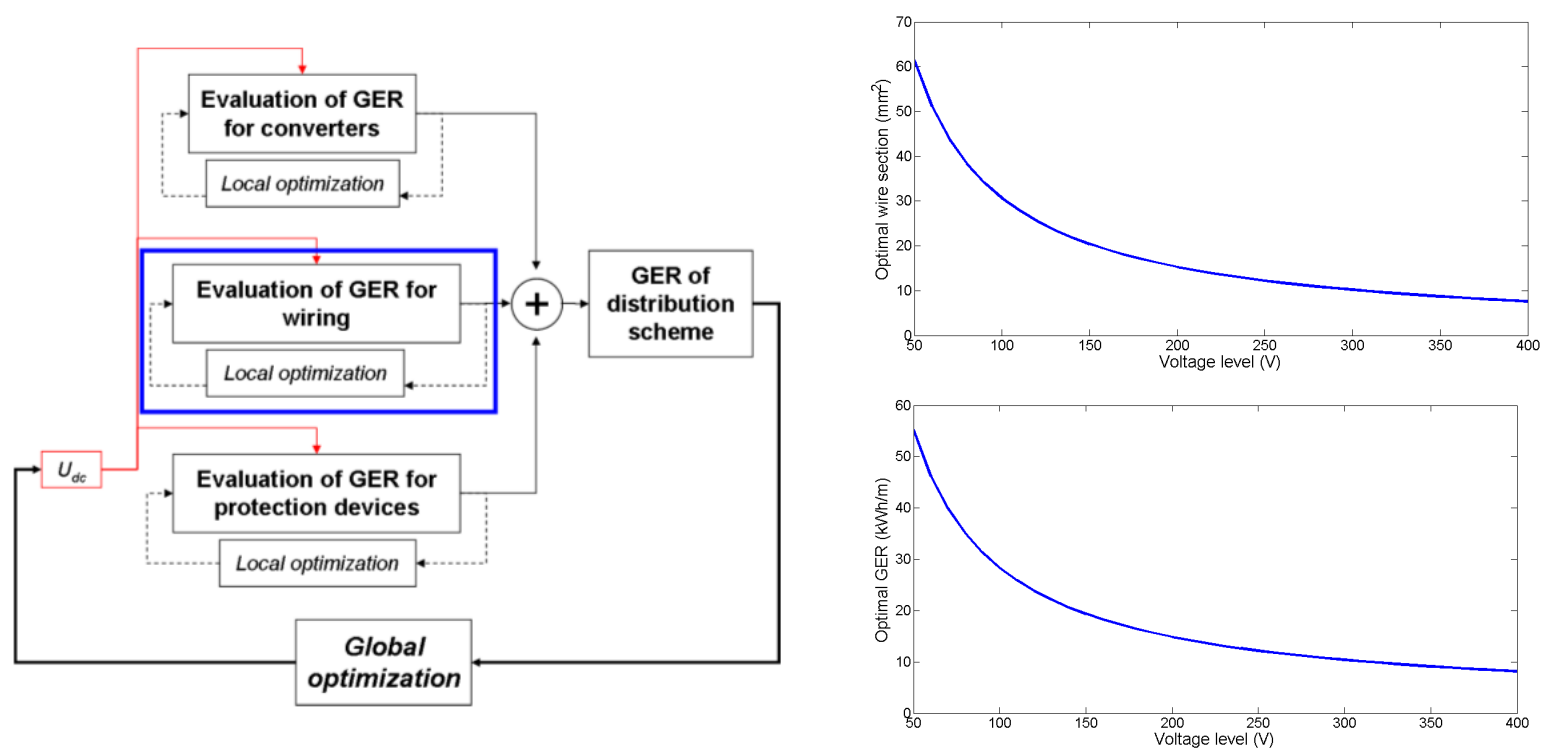

Figure 8 : optimisation du dimensionnement du système (câblage et convertisseurs statiques) de distribution DC

[JAO11]. A gauche : organigramme. A droite : section et GER optimaux en fonction du niveau de tension.

Optimization design DC distribution system (wiring, static converters) [JAO11]. Left: organization chart. Right: optimal section and GER versus voltage level.

Nous avons donc dû (laborieusement) produire des données initialement inexistantes sur les différents composants pour pouvoir mettre en place des procédures d'optimisation sur cycle de vie. La procédure globale est 
décrite sur la figure 8a (issue de la référence [JAO11] en cours de reviewing) tandis que la figure $8 \mathrm{~b}$ montre un résultat d'optimisation du GER du câblage, pour répondre à un profil de consommation particulier.

\section{V-Conclusion}

L'éco-conception des systèmes électriques constitue un très vaste champ de recherche dans lequel nous n'avons apporté qu'une modeste contribution en abordant le problème du point de vue de l'éco-dimensionnement, et selon des critères très restrictifs, à savoir essentiellement l'énergie primaire consommée sur l'ensemble du cycle de vie. Nous avons fait ce choix pour nous affranchir de la complexité associée à la multitude des critères environnementaux et sachant que l'énergie primaire, en tout cas dans les dispositifs électriques, possède un poids majeur, surtout lorsqu'elle est d'origine non renouvelable. Cette approche permet également de ne pas accorder une importance excessive au carbone, ce qui a tendance à faire oublier que l'énergie nucléaire et même les énergies renouvelables ont également des impacts environnementaux parfois très significatifs ou encore mal évalués. A travers quelques exemples, nous avons montré l'étendue des applications et des approches, nous avons également très rapidement survolé les difficultés importantes associées à la recherche de modélisations et de méthodes d'optimisation adaptées à la résolution de ces problèmes lourds ainsi qu'à la nécessité de modèles d'évaluation de la durée de vie.

Nous pensons que l'introduction de critères environnementaux dans la conception des systèmes électrotechniques est une nécessité, plus particulièrement encore si l'on considère que l'électricité offre la capacité d'être un vecteur essentiel du développement durable grâce à ses nombreuses qualités (facilité à convertir les ressources énergétiques renouvelables, possibilités de hauts rendements de conversion, grande finesse de réglage...). S'ouvre ainsi un vaste champ de recherche auquel nous invitons tous nos collègues à contribuer.

Afin d'accélérer la pénétration des modes de raisonnement sur cycle de vie et, entre autres, de la compréhension des notions d'impacts environnementaux, il nous semble également fondamental de les intégrer rapidement à tous les niveaux de formation, nous avons d'ailleurs présenté récemment une communication sur ce sujet [DEB10a] afin de faire connaître notre expérience en la matière à l'ENS de Cachan sur le site de Bretagne en filière mécatronique.

\section{$\underline{\text { VI-Bibliographie }}$}

[AUB10a] J. AUBRY, H. BEN AHMED, B. MULTON, « Bi-Objective Sizing Optimization of a PM Machine Drive on an Operating Profile », ICEM 2010 (Rome), 6-9 oct. 2010, 7 p.

[AUB10b] J. AUBRY, P. BYDLOWSKI, B. MULTON, H. BEN AHMED, B. BORGARINO, « Energy Storage System Sizing for Smoothing Power Generation of Direct Wave Energy Converters », 3rd Intern. Conf. on Ocean Energy (ICOE), Bilbao, 6 oct. 2010.

[DEB09] V. DEBUSSCHERE, «Contributions méthodologiques à l'éco-conception de convertisseurs électromagnétiques d'énergie », thèse de doctorat de l'ENS de Cachan, soutenue le 9 décembre 2009, http://tel.archives-ouvertes.fr/tel-00443702_v3/

[DEB10a] V. DEBUSSCHERE, B. MULTON, H. BEN AHMED, C. RICORDEL, M.D.- de CAYEUX, P. GOURRONC, Y. LEGUERN, « Enseignement en Eco-Conception : une expérience à l'ENS de Cachan », Conf. CETSIS 2010, Grenoble, 2010, 10p.

[DEB10b] V. DEBUSSCHERE, B. MULTON, H. BEN AHMED, P.E. CAVAREC, « Life cycle design of a single-phase induction motor », Electric Power Applications IET, Vol. 4 n5, may 2010, pp.348 - 356.

[DIA06] Y. DIAB, P. VENET, G. ROJAT, "Comparison of the Different Circuits Used for Balancing the Voltage of Supercapacitors: Studying Performance and Lifetime of Supercapacitors" ESSCAP, Lausanne, 2006.

[CRET05] P. CRETTAZ, M. SAADÉ, O. JOLLIET, « Analyse du cycle de vie - Comprendre et réaliser un écobilan », Presses Polytechniques et Universitaires Romandes, 2005.

[IEA10] International Energy Agency, 2010 Key World Energy Statistics, C OECD/IEA, 2010.

[JAO11] C. JAOUEN, B. MULTON, F. BARRUEL, "Wiring design based on Global Energy Requirement criteria: a first step towards an eco-designed DC distribution scheme”, soumis à IREED 2011, Lille 23-24 March 2011.

[LIS10] J. LISCOUËT, M. BUDINGER, J.C. MARÉ, « Design for Reliability of Electromechanical Actuators », Recent Advances in Aerospace Actuation Systems and Components, May 5-7 2010, Toulouse, 9 p.

[MUL08] B. MULTON, H. BEN AHMED, V. DEBUSSCHERE, J. SEIGNEURBIEUX, M. RUELLAN, Y. THIAUX, «Ecoconception : de nouveaux critères de conception en électrotechnique pour le développement durable », REE n 5 , mai 2008, pp89-98.

[OBS09] Observ'ER, La production d'électricité d'origine renouvelable dans le monde, $11^{\text {ème }}$ inventaire, 2009.

[THI10a] Y. THIAUX, J. SEIGNEURBIEUX, B. MULTON, H. BEN AHMED, « Load Profile Impact on the Gross Energy Requirement of Stand-Alone Photovoltaic System », Renewable Energy Journal Elsevier, n³5 (2010) pp.602-613.

[THI10b] Y. THIAUX, «Optimisation des profils de consommation pour minimiser les coûts économique et énergétique sur cycle de vie des systèmes photovoltaïques hybrides. ", thèse de l'ENS de Cachan, 2010, http://tel.archives-ouvertes.fr/tel-00502428/en/

[VENT97] J.P. VENTERE, « Conception écologique des produits », Les Techniques de l'Ingénieur, G 6000, 1997. 